\title{
Estudo de Validação e Fidedignidade de Escalas de Silhuetas Brasileiras em Adolescentes
}

\author{
Maria Fernanda Laus \\ Sebastião Sousa Almeida ${ }^{1}$ \\ Universidade de São Paulo \\ Mariana Barticiotti Murarole \\ Telma Maria Braga-Costa \\ Universidade de Ribeirão Preto
}

\begin{abstract}
RESUMO - Objetivou-se avaliar a validade de construto e a fidedignidade das escalas de silhuetas de Kakeshita (2008) em adolescentes brasileiros. Após a condução do teste-reteste em 112 participantes com intervalo de um mês, os resultados demonstraram que as escalas foram capazes de discriminar os diferentes grupos de estado nutricional em ambos os sexos; apresentaram coeficientes de correlação entre IMC real, IMC atual e insatisfação e coeficientes de correlação intraclasse elevados, comprovando que o instrumento é apropriado para avaliar a imagem corporal nessa faixa etária.
\end{abstract}

Palavras-chave: imagem corporal, escalas de silhuetas, fidedignidade, validade, adolescentes

\section{Validation and Reliability Study of the Figure Rating Scales Applied to Brazilian Adolescents}

\begin{abstract}
This study aimed to obtain evidence of the construct validity and the reliability of the Figure Rating Scales developed by Kakeshita (2008) applied to Brazilian adolescents. After conducting the test-retest in 112 participants with an interval of one month, the results showed that the scales were able to discriminate the different groups of nutritional status in both sexes; showed significant correlation between current BMI, desired BMI and dissatisfaction; and coefficients of elevated intraclass correlation, indicating that the instrument is suitable for assessing body image in this age group.
\end{abstract}

Keywords: body image, figure rating scale, reliability, validity, adolescents

A imagem corporal é definida como as percepções, pensamentos e sentimentos de um indivíduo a respeito de seu próprio corpo (Grogan, 2008) e inclui uma dimensão perceptual e uma atitudinal. A dimensão perceptual refere-se à acurácia do julgamento de um indivíduo sobre seu tamanho, formato e peso relativos às suas proporções atuais (Cash, Wood, Phelps, \& Boyd, 1991), sendo geralmente medida por meio do grau de distorção entre o tamanho do corpo real e o percebido (Cash, 2012). Os distúrbios na percepção da imagem corporal resultam em uma sub ou superestimação do corpo como um todo ou partes dele, e a já extensamente conhecida relação entre essas distorções e os distúrbios alimentares reforçam a necessidade de investigação dessa dimensão (Gardner, 2011).

Já a dimensão atitudinal é composta por quatro componentes: afetivo, cognitivo, comportamental e insatisfação global (Menzel, Krawczyk, \& Thompson, 2011). Este último pode ser ainda definido como "os pensamentos e sentimentos negativos de uma pessoa sobre seu corpo" (Grogan, 2008, p. 4), substancialmente derivados da discrepância entre a aparência autopercebida e o corpo considerado ideal pelo indivíduo (Cash, 2011), sendo um grande preditor de com-

1 Endereço para correspondência: Departamento de Psicologia, Faculdade de Filosofia, Ciências e Letras de Ribeirão Preto, Universidade de São Paulo, Av. Bandeirantes, 3900, Monte Alegre, CEP 14040901, Ribeirão Preto, SP, Brasil.E-mail: sebasalm@usp.br portamentos de risco à saúde, como baixa autoestima (Berg, Mond, Eisenberg, Ackard, \& Neumark-Sztainer, 2010), sintomas depressivos (Braush \& Gutierrez, 2009), ideais suicidas (Crow, Eisenberg, Story, \& Neumark-Sztaine, 2008), comportamento alimentar inadequado e desenvolvimento de transtornos alimentares (Westerber-Jacobson, Edlund, \& Ghaderi, 2010).

Entre os métodos disponíveis atualmente para a avaliação desse componente destacam-se dois - os questionários e as escalas de silhuetas. Os questionários são capazes de acessar diferentes focos e características do construto simultaneamente, por meio de escalas de respostas tipo Likert ou analógica visual, e alguns tiveram pontos de cortes estabelecidos em base populacional para determinar distúrbios clinicamente significativos (Menzel et al., 2011). Entretanto, esses instrumentos são, em geral, demasiadamente longos e exigem alto grau de literalidade dos participantes. Assim, as escalas de figuras de silhuetas constituem-se uma das medidas mais utilizadas ao redor do mundo para aferição da insatisfação com o tamanho do corpo (Kakeshita, Laus, \& Almeida, 2013).

Além de configurarem-se instrumentos relativamente simples e de baixo custo, por não exigirem equipamentos sofisticados, são aplicados e manuseados de maneira fácil e rápida, podem ser empregados em grandes números amostrais e não demandam fluência verbal dos participantes ou diversidade de vocabulário (Gardner \& Brown, 2010). Essas 
escalas são, em geral, constituídas por contornos de silhuetas do corpo humano, em uma variedade de tamanhos, pesos e formatos, desde desenhos muito magros a desenhos muito obesos (Menzel et al., 2011). Segundo Thompson, Burke e Krawczyk (2012), habitualmente, o participante deve indicar a figura que melhor representa seu tamanho atual (medida que reflete seu corpo percebido) e a figura que melhor representa o corpo que considera ideal (também chamado de desejado). A diferença entre essas escolhas é tida como indicativa de algum grau de insatisfação (ex. Adami et al., 2012; Conti \& Latorre, 2009; Gardner, Jappe, \& Gardner, 2009; Gardner, Stark, Jackson, \& Friedman, 1999; Kakeshita, 2008; Stunkard, Sørensen, \& Schulsinger, 1983; Thompson \& Gray, 1995).

Há que se considerar, porém, que inúmeros problemas têm sido identificados nas escalas existentes, sendo os mais comuns: representações irreais do corpo humano; desproporções entre os membros superiores e inferiores, direitos e esquerdos; desenhos com características faciais e corporais que refletem apenas a etnia caucasiana; pequeno número de figuras, geralmente entre sete e nove para cada sexo; incrementos não constantes nos intervalos entre as figuras adjacentes; apresentação em uma única folha de papel; natureza ordinal das figuras; impossibilidade de aferição do componente perceptual da imagem corporal; e abstenção de medidas de validade e fidedignidade (Gardner, Friedman, \& Jackson, 1998; Gardner \& Brown, 2010).

Diante disso, merecem destaque as escalas construídas por Kakeshita (2008), que foram desenvolvidas com base nas medidas antropométricas médias de homens e mulheres brasileiros e são constituídas por 15 cartões plastificados para cada sexo. Neles, cada figura corresponde a um Índice de Massa Corporal (IMC) - medida que expressa o peso $(\mathrm{kg})$ dividido pelo quadrado da altura (m) -, variando de 12,5 a $47,5 \mathrm{~kg} / \mathrm{m}^{2}$ e com diferença constante de $2,5 \mathrm{~kg} / \mathrm{m}^{2}$ entre as figuras. No estudo de fidedignidade teste-reteste (com um mês de intervalo) conduzido em 46 mulheres e 44 homens, a autora encontrou coeficientes de correlação superiores a 0,70 para as escalas feminina e masculina $(p<0,01)$. As figuras foram corretamente ordenadas por mais de $83 \%$ dos participantes, sendo os erros cometidos sempre entre figuras adjacentes, o que se justifica pelas características psicofísicas das escalas que preveem uma diferença mínima perceptível entre as figuras (Kakeshita, Silva, Zanatta, \& Almeida, 2009).

Sua construção buscou atender rigorosamente as recomendações metodológicas propostas por estudiosos renomados (Gardner et al., 1998; 1999; Thompson \& Gray, 1995), sendo desenvolvidas com um maior número de figuras que as demais, garantindo boa abrangência; desenhadas sem detalhes fisionômicos que pudessem atuar como distratores da percepção visual, porém com representações realistas da silhueta humana e formas características suficientes para a identificação de cada sexo; com diferenças mínimas perceptíveis e constantes nas medidas entre figuras adjacentes; e apresentadas em conjunto de cartões plastificados individuais.

Tradicionalmente as escalas de silhuetas acessam exclusivamente a insatisfação (Banitt et al., 2008; Chen, Fox, Haase, \& Ku, 2010; Dumith et al., 2012; Gray, Simon, Janicke, \& Dumont-Driscoll, 2011; Laus, Braga Costa, \&
Almeida, 2011; Torresani et al., 2009; Xie et al., 2010), porém, adicionalmente às escalas desenvolvidas por Gardner $\mathrm{e}$ colaboradores (2009), as de Kakeshita (2008) constituem-se as únicas capazes de mensurar também a acurácia da estimação do tamanho corporal real. Isso porque, entre todas as escalas disponíveis atualmente, apenas essas duas utilizaram dimensões antropométricas conhecidas de indivíduos com diferentes IMC para gerar cada uma das figuras. Não obstante uma variedade de técnicas tenha sido desenvolvida para acessar essa dimensão, incluindo métodos computadorizados de vídeo distorção, esse tipo de metodologia requer que os dados sejam coletados individualmente, tem alto custo, consome muito tempo e é cansativo para os participantes (Gardner, 2012), o que confere às escalas vantagens significativas em comparação a ele.

Assim, nas escalas de Kakeshita (2008), cada figura tem correspondência a um valor médio e intervalos de IMC pré-determinados, o que possibilita a análise dos resultados de estimação corporal e insatisfação corporal também em termos numéricos, facilitando análises quantitativas dos dados, o que é uma limitação dos outros instrumentos disponíveis na literatura para o estudo da imagem corporal.

No Brasil, Laus et al. (2013) afirmam que os adolescentes correspondem à faixa etária com o maior número de publicações sobre o tema (p. ex. Adami, Frainer, Santos, Fernandes, \& De-Oliveira, 2008; Branco, Hilário, \& Cintra, 2006; Corseuil, Pelegrini, Beck, \& Petroski, 2009; Dumith et al., 2012; Pereira, Graup, Lopes, Borgato, \& Daronco, 2009), embora haja, notadamente, uma escassez de instrumentos desenvolvidos especificamente para avaliação da imagem corporal nessa faixa etária em todo o mundo (para maiores detalhes ver Menzel et al., 2011 e Thompson et al., 2012). Um levantamento recente a respeito dos instrumentos disponíveis no país (Laus et al., 2013) demonstrou ausência de medidas capazes de acessar a dimensão perceptiva na população adolescente e existência de três questionários: a Body Area Scale - BAS (Conti, Latorre, Hearst, \& Segurado, 2009), a Escala de Evaluación de Insatisfación Corporal (Conti, Slater, \& Latorre, 2009) e o Body Shape Questionnaire - BSQ (Conti, Cordás, \& Latorre, 2009) e de duas escalas de silhuetas capazes de aferir a insatisfação corporal. O estudo de Conti e Latorre (2009) explorou a validade de construto e a reprodutibilidade da escala de nove figuras de Thompson e Gray (1995), enquanto Adami et al. (2012) testaram a validade de construto da escala de oito figuras de Childress, Brewerton, Hodges e Jarrell (1993). Embora essas duas escalas tenham apresentado índices razoáveis de correlação, ambas possuem algumas das mais importantes limitações apontadas por Gardner et al. (1998).

Recentemente, Cortês, Meireles, Friche, Caiaffa e Xavier (2013) publicaram uma revisão sistemática da literatura a respeito do uso de escalas de silhuetas na avaliação da satisfação corporal de adolescentes e concluíram que a escala mais utilizada foi a de Stunkard et al. (1983), inclusive no Brasil, e que, em sua maioria, esses trabalhos apresentam limitações importantes no que se refere à análise dos dados e à forma de apresentação do instrumento. Além disso, vale ressaltar que essa escala teve sua validade concorrente e discriminante testada em mulheres brasileiras por Scagliusi et al. (2006), mas não em adolescentes. 
Considerando que em pesquisas que envolvem imagem corporal a utilização de medidas que não tenham sua fidedignidade e validade devidamente testadas certamente prejudica a confiabilidade dos dados, e que não há no Brasil escalas de silhuetas desenvolvidas especificamente para acessar a imagem corporal de adolescentes, faz-se necessário o empreendimento de esforços para desenvolver/adaptar medidas adequadas. Uma vez que as escalas criadas por Kakeshita (2008) levam em consideração o biotipo da população brasileira e apresentam as já citadas inúmeras vantagens em relação às escalas existentes, o presente estudo teve por objetivo testar a validade de construto e a fidedignidade dessas escalas em adolescentes no país.

\section{Método}

\section{Participantes}

Trata-se de uma pesquisa de corte transversal, do tipo metodológica, em uma amostra de conveniência. $\mathrm{O}$ estudo contou com a participação de 118 adolescentes (58 meninos e 60 meninas) na fase de teste (primeira aplicação do instrumento), entretanto, seis deles não responderam ao reteste, o que resultou em uma amostra final constituída por 112 adolescentes voluntários ( 55 meninos e 57 meninas), entre 14 e 17 anos. Desses, 37 meninos e 46 meninas eram provenientes de uma escola pública de Ribeirão Preto, enquanto 18 meninos e 11 meninas de uma escola particular de Vargem Grande do Sul. Entre o sexo masculino, as médias $( \pm D P)$ de idade e IMC foram respectivamente: 15,49 anos $( \pm 0,74) \mathrm{e}$ $22,03 \mathrm{~kg} / \mathrm{m}^{2}( \pm 3,66)$. Já entre o sexo feminino estas médias foram iguais a 15,38 anos $( \pm 0,72)$ e $21,22 \mathrm{~kg} / \mathrm{m}^{2}( \pm 2,92)$.

\section{Procedimento}

Antes do inicio do estudo, os pesquisadores entraram em contato com a diretoria das escolas e, obtidas as autorizações, os alunos foram abordados em sala de aula, expostos à natureza da pesquisa e solicitados a entregar aos pais o Termo de Consentimento Livre e Esclarecido. Obtido o consentimento dos responsáveis e dos adolescentes, a coleta de dados ocorreu em salas cedidas pelas próprias instituições.

Na primeira etapa do estudo (teste), os adolescentes foram, individualmente, apresentados às escalas de silhuetas, dispostas em séries ordenadas ascendentes. Para a avaliação da acurácia da estimação do tamanho corporal, os participantes deveriam escolher a figura que melhor representasse seu tamanho corporal atual (IMC Atual). Já a avaliação da (in) satisfação foi acessada por meio da solicitação da escolha da figura que melhor representasse o tamanho que gostariam de ter (IMC Desejado). Os dados foram tabulados e classificados por sexo. Foi calculado o IMC real e registrados os IMCs escolhidos por cada participante, de acordo com o protocolo experimental. A acurácia da estimação do tamanho corporal foi avaliada subtraindo-se o IMC Real do IMC escolhido como "Atual" (IMC Atual - IMC Real) e a insatisfação foi medida através da discrepância entre o IMC "Desejado" e o
IMC “Atual” (IMC Desejado - IMC Atual). Nesse caso, os resultados foram dados em forma de médias ( $\pm \mathrm{DP}$ ). Quanto mais próxima de zero, mais acurada foi a estimação e menor a insatisfação. Resultados negativos indicavam uma subestimação do tamanho corporal real e um anseio por uma silhueta menor, enquanto resultados positivos indicavam superestimação e um desejo por uma silhueta maior.

Em seguida, cada participante teve seu peso e altura aferidos. O peso corporal foi medido utilizando-se uma balança de plataforma portátil, da marca Tanita ${ }^{\circledR}$ (carga máxima de 150 $\mathrm{kg}$ e precisão de $100 \mathrm{~g}$ ), com os adolescentes posicionados em pé, descalços e trajando roupas leves. A estatura foi aferida com um estadiômetro portátil fixado verticalmente em uma parede, com os participantes também descalços, com os pés paralelos e tornozelos unidos; as nádegas, os ombros e a parte posterior da cabeça encostados em uma parede, estando os braços soltos ao longo do corpo. $\mathrm{O}$ adolescente permaneceu em pé, ereto, sem encolher ou esticar, olhando para frente e a leitura foi efetuada o mais próximo de $0,5 \mathrm{~cm}$. Os dados de peso e altura foram inseridos no software EpiInfo ${ }^{\circledR}$ para obtenção de percentis de IMC. Posteriormente, o estado nutricional foi classificado através de percentis específicos para sexo e idade, baseados nos parâmetros recomendados pelo Ministério da Saúde (2007) que classifica cada indivíduo de acordo com os limites de percentis: baixo peso $\mathrm{P}<3$; eutrofia $\geq \mathrm{P} 3$ e $\mathrm{P}<85$; sobrepeso $85 \leq \mathrm{P}<97$, e obesidade $\mathrm{P}$ $\geq 97$. É importante ressaltar que, uma vez que apenas quatro meninos e uma menina foram classificados com obesidade, optou-se por incluí-los no grupo sobrepeso, que foi nomeado de grupo "acima do peso".

Seguindo os procedimentos realizados por Kakeshita (2008) para avaliação da fidedignidade das escalas originais em adultos, os procedimentos relativos ao reteste foram repetidos com um intervalo de um mês a partir da data da primeira aplicação.

\section{Análise dos dados}

Foi testada a distribuição dos dados por meio do Teste de Kolmogorov-Smirnov. Uma vez que os dados não apresentaram uma distribuição normal, foram utilizados testes não-paramétricos. A avaliação da validade de construto foi conduzida por meio do teste de Kruskal Wallis, comparando os três grupos de estado nutricional (baixo peso, eutrofia e acima do peso) e do coeficiente de correlação de Spearman (IMC real x IMC Atual x insatisfação). Já a fidedignidade teste-reteste foi calculada utilizando um teste de correlação intraclasse (CCI) e pela comparação das médias dos resultados da escala no teste e no reteste por meio do teste de Wilcoxon. Todas as análises foram realizadas utilizando o software SPSS para Windows, versão 17.0.

\section{Resultados}

A Tabela 1 apresenta as médias de insatisfação e os resultados do teste de Kruskal Wallis na análise da validade de construto das escalas, por sexo e estado nutricional. Presume-se que, para que o instrumento seja considerado 
Tabela 1. Resultados do teste de Kruskal Wallis na análise da validade de construto das escalas de silhuetas, por sexo e estado nutricional.

\begin{tabular}{lcccc}
\hline Categoria & n & Média & DP & p \\
\hline Masculino & & & & \\
$\quad$ Baixo peso & 09 & 2,78 & 4,58 & \\
$\quad$ Eutrofia & 36 & $-1,25$ & 4,37 & $<0,01$ \\
$\quad$ Sobrepeso & 10 & $-5,00$ & 6,87 & \\
\hline Feminino & & & & \\
$\quad$ Baixo peso & 07 & 2,86 & 3,03 & \\
$\quad$ Eutrofia & 44 & $-3,30$ & 3,84 & $<0,001$ \\
$\quad$ Sobrepeso & 06 & $-8,75$ & 1,36 & \\
\hline
\end{tabular}

Nota: $\mathrm{DP}=$ desvio padrão

válido, a insatisfação seja proeminente entre meninos e meninas acima do peso e, conforme esperado, nossos resultados demonstraram que houve uma diferença estatisticamente significativa nas médias de insatisfação entre os grupos, em ambos os sexos.

As análises de correlação de Spearman, também conduzidas para avaliação da validade de construto, entre o IMC real dos adolescentes e os resultados das escalas, por sexo, estão descritas na Tabela 2. Supondo que quanto maior o IMC real maiores são o IMC escolhido como atual e a insatisfação com o tamanho do corpo, os dados demonstraram resultados satisfatórios, com correlações estatisticamente significativas para todas as análises, em ambos os sexos.

Já os resultados das análises de fidedignidade encontram-se na Tabela 3. Os coeficientes de correlação intraclasse (CCI) para IMC atual, IMC desejado, acurácia da estimação e insatisfação com o tamanho do corpo foram positivos e significativos para meninos e meninas, sendo superiores a 0,95 $(p<0,001)$ em todas as análises. Além disso, por meio do
Tabela 2. Coeficiente de correlação de Spearman na avaliação da validade de construto das escalas de silhuetas, por sexo.

\begin{tabular}{lccc}
\hline Variável & IMC Real & IMC Atual & Insatisfação \\
\hline Masculino & & & \\
IMC Real & 1,00 & $0,62^{*}$ & $-0,44^{*}$ \\
IMC Atual & - & 1,00 & $-0,78^{*}$ \\
Insatisfação & - & - & 1,00 \\
\hline Feminino & & & \\
IMC Real & 1,00 & $0,46^{*}$ & $-0,53^{*}$ \\
IMC Atual & - & 1,00 & $-0,69^{*}$ \\
Insatisfação & - & - & 1,00 \\
\hline
\end{tabular}

Nota: ${ }^{*} \mathrm{p}<0,001$

teste de Wilcoxon, as médias observadas entre os resultados do teste e do reteste para as meninas em todas as variáveis não foram diferentes estatisticamente, o mesmo não sendo observado para os meninos em relação ao IMC desejado e a insatisfação (Tabela 3).

\section{Discussão}

As escalas de silhuetas propostas por Kakeshita (2008) apresentaram resultados muito satisfatórios na avaliação da validade de construto e da fidedignidade teste-reteste em adolescentes. Esses dados são de extrema importância para as pesquisas sobre imagem corporal no país, uma vez que é consenso que uma das principais preocupações no uso desse tipo de escala nessa população específica envolve a insuficiência de estudos relativos às qualidades psicométricas dos instrumentos utilizados (Smolak, 2004; Thompson \& Berg, 2002).

Tabela 3. Resultados da avaliação da fidedignidade das escalas de silhuetas, por sexo

\begin{tabular}{|c|c|c|c|c|c|c|c|c|}
\hline \multirow{2}{*}{ Variável } & \multicolumn{4}{|c|}{ Masculino } & \multicolumn{4}{|c|}{ Feminino } \\
\hline & Média & $\mathrm{DP}$ & $\mathrm{CCI}$ & Teste de Wilcoxon & Média & DP & $\mathrm{CCI}$ & Teste de Wilcoxon \\
\hline \multicolumn{9}{|c|}{ IMC Atual } \\
\hline Teste & 23,00 & 5,76 & \multirow{2}{*}{$0,99 *$} & \multirow{2}{*}{ ns } & 25,21 & 5,59 & \multirow{2}{*}{$0,99 *$} & \multirow{2}{*}{ ns } \\
\hline Reteste & 23,00 & 5,70 & & & 25,30 & 5,69 & & \\
\hline \multicolumn{9}{|c|}{ IMC Desejado } \\
\hline Teste & 21,72 & 3,69 & \multirow{2}{*}{$0,95^{*}$} & \multirow{2}{*}{$<0,01$} & 22,10 & 4,16 & \multirow{2}{*}{$0,96^{*}$} & \multirow{2}{*}{ ns } \\
\hline Reteste & 21,18 & 3,14 & & & 21,71 & 3,81 & & \\
\hline \multicolumn{9}{|c|}{ Estimação } \\
\hline Teste & 0,96 & 4,61 & \multirow{2}{*}{$0,99 *$} & \multirow{2}{*}{ ns } & 3,99 & 5,01 & \multirow{2}{*}{$0,98 *$} & \multirow{2}{*}{ ns } \\
\hline Reteste & 0,96 & 4,59 & & & 4,08 & 4,89 & & \\
\hline \multicolumn{9}{|c|}{ Insatisfação } \\
\hline Teste & $-1,27$ & 5,35 & \multirow{2}{*}{$0,98 *$} & \multirow{2}{*}{$<0,01$} & $-3,11$ & 4,51 & \multirow{2}{*}{$0,96^{*}$} & \multirow{2}{*}{$\mathrm{ns}$} \\
\hline Reteste & $-1,81$ & 5,14 & & & $-3,59$ & 5,17 & & \\
\hline
\end{tabular}

Notas: $\mathrm{DP}=$ desvio padrão; $\mathrm{CCI}=$ coeficiente de correlação intraclasse; $\mathrm{ns}=$ não significativo. ${ }^{*} \mathrm{p}<0,001$ 
Conforme esperado, as escalas de Kakeshita (2008) foram capazes de discriminar adequadamente os diferentes grupos de estado nutricional e apresentaram índices de correlações significativos em todas as análises em ambos os sexos. Na avaliação da fidedignidade, as escalas apresentaram excelentes índices de estabilidade temporal, atestadas por meio dos coeficientes de correlação intraclasse superiores a 0,90 em meninos e meninas, estando, portanto, acima do valor mínimo recomendado por Thompson (2004) para que um instrumento possa ser considerado fidedigno $(0,70)$.

No Brasil, conforme já citado, apenas duas escalas de silhuetas tiveram suas qualidades psicométricas testadas em adolescentes. No estudo de Adami et al. (2012) sobre validade de construto, as escalas de Childress et al. (1993) apresentaram coeficientes de correlação entre o IMC real e a figura escolhida como atual iguais a $0,62(p<0,001)$ para os meninos e $0,54(p<0,001)$ para as meninas. Já os coeficientes de correlação entre o IMC real e a insatisfação corporal foram de $0,37(p<0,001)$ para o sexo feminino e $-0,01$ $(p>0,9)$ para o masculino. Conti e Latorre (2009) também avaliaram a validade de construto e encontraram correlações estatisticamente significativas nas análises entre o escore das escalas de Thompson e Gray (1995) e o IMC real para os meninos $(-0,61 ; p<0,001)$ e meninas $(-0,52 ; p<0,001)$ e não observaram diferença estatisticamente significativa nas médias dos escores do teste e do reteste apenas entre o sexo masculino. Ainda, na avaliação da fidedignidade, os coeficientes de correlação intraclasse entre os escores dos dois momentos foram significativos para os meninos $(0,86 ; p<0,001)$ e meninas $(0,80 ; p<0,001)$ (Conti \& Latorre, 2009).

Note que, embora os valores de correlação encontrados no presente estudo sejam similares aos reportados por Adami et al. (2012) e Conti e Latorre (2009) no que se refere à validade de construto, tanto as escalas de Childress et al. (1993) quanto as de Thompson e Gray (1995) apresentam limitações importantes: número restrito de figuras, inconstância no incremento entre cada uma das silhuetas e incapacidade de mensuração do componente perceptual da imagem corporal (Gardner et al., 1998). Além disso, nenhuma delas foi desenvolvida com base no biotipo da população brasileira, o que pode acarretar críticas importantes ao seu uso já que em estudos sobre imagem corporal é essencial que se escolha instrumentos apropriados para a população alvo, levando em conta a idade, o sexo e aspectos culturais dos participantes que serão estudados (Cash, 2011; Thompson, 2004).

Apesar de todas as suas vantagens, as escalas de Kakeshita (2008) também apresentam algumas limitações. Primeiramente, elas levam em consideração apenas o tamanho da silhueta, relativo à magreza/obesidade, mas não contemplam as proporções relativas a partes específicas do corpo, como quadril, bustos, coxas, peito. Ademais, as escalas não levam em conta a questão da muscularidade, especialmente importante na avaliação do sexo masculino.

Assim como as escalas em si, o presente estudo também apresenta algumas limitações: 1) falta de informações demográficas a respeito da amostra, que impossibilita que nossos dados sejam generalizados para a população de adolescentes no país; 2) pequeno número amostral e 3) resultados que se configuram análises preliminares do processo de adaptação, havendo necessidade de estender não apenas o tamanho da amostra, mas também os tipos de validade (de conteúdo e de critério).

Em conclusão, a aplicação das escalas de silhuetas desenvolvidas por Kakeshita (2008) em adolescentes produziu ótimos indícios de validade e fidedignidade. Por todas as vantagens que essas escalas apresentam em relação às demais, é possível considerá-las superiores para avaliação dessa faixa etária e, portanto, o instrumento mais indicado para a população brasileira.

\section{Referências}

Adami, F., Frainer, D. E. S., Almeida, F. S., Abreu, L. C., Valenti, V. E., Demarzo, M. M. P., Monteiro, C. B. M., \& Oliveira, F. R. (2012). Construct validity of a figure rating scale for Brazilian adolescents. Nutrition Journal, 11(1), 24. doi:10.1186/14752891-11-24

Adami, F., Frainer, D. E. S., Santos, J. S., Fernandes, T. C., \& De-Oliveira, F. R. (2008). Insatisfação corporal e atividade física em adolescentes da região continental de Florianópolis. Psicologia: Teoria e Pesquisa, 24(2), 143-149. doi:10.1590/ S0102-37722008000200003

Banitt, A. A., Kaur, H., Pulvers, K. M., Nollen, N. L., Ireland, M., $\&$ Fitzgibbon, M. L. (2008). BMI percentiles and body image discrepancy in black and white adolescents. Obesity, 16(5), 987-991. doi:10.1038/oby.2008.21

Berg, P. A. van den, Mond, J., Eisenberg, M., Ackard, D., \& Neumark-Sztainer, D. (2010). The link between body dissatisfaction and self-esteem in adolescents: similarities across gender, age, weight status, race/ethnicity, and socioeconomic status. Journal of Adolescent Health, 47(3), 290-296. doi:10.1016/j.jadohealth.2010.02.004

Branco, L. M., Hilário, M. O. E., \& Cintra, I. P. (2006). Percepção e satisfação corporal em adolescentes e a relação com seu estado nutricional. Revista de Psiquiatria Clínica, 33(6), 292-296. doi:10.1590/S0101-60832006000600001

Brausch, A. M., \& Gutierrez, P. M. (2009). The role of body image and disordered eating as risk factors for depression and suicidal ideation in adolescents. Suicide and Life-Threatening Behavior, 39(1), 58-71. doi:10.1521/suli.2009.39.1.58

Cash, T. F. (2011). Cognitive-behavioral perspectives on body image. In T. F. Cash, \& L. Smolak (Eds.), Body image: a handbook of science, practice, and prevention (pp. 39-47). New York: The Guilford Press.

Cash, T. F. (2012). Cognitive-behavioral perspectives on body image. In T. F. Cash (Ed.). Encyclopedia of body image and human appearance (pp. 334-342). Oxford: Elsevier.

Cash, T. F., Wood, K. C., Phelps, K. D., \& Boyd, K. (1991). New assessments of weight-related body image derived from extant instruments. Perceptual and Motor Skills, 73(1), 235-241. doi:10.2466/pms.1991.73.1.235

Chen, L. J., Fox, K. R., Haase, A., \& Ku, P. W. (2010). Correlates of body dissatisfaction among Taiwanese adolescents. Asia Pacific Journal of Clinical Nutrition, 19(2), 172-179. Retrieved from http://211.76.170.15/server/APJCN/Volume19/vol19.2/ Finished/3_1604_172-179.pdf 
Childress, A. C., Brewerton, T. D., Hodges, E. L., \& Jarrell, M. P. (1993). The Kids' Eating Disorders Survey (KEDS): a study of middle school students. Journal of the American Academy of Child \& Adolescent Psychiatry, 32(4), 843-850. doi: 10.1097/00004583-199307000-00021

Conti, M. A., \& Latorre, M. R. D. O. (2009). Estudo de validação e reprodutibilidade de uma escala de silhueta para adolescentes. Psicologia em Estudo, 14(4), 699-706. doi:10.1590/S141373722009000400010

Conti, M. A., Latorre, M. R. D. O., Hearst, N., \& Segurado, A. (2009). Cross-cultural adaptation, validation and reliability of the Body Area Scale for Brazilian adolescents. Cadernos de Saúde Pública, 25(10), 2179-2186. doi:10.1590/S0102311X2009001000009

Conti, M. A., Slater, B., \& Latorre, M. R. D. O. (2009). Validação e reprodutibilidade da Escala de Evaluación de Insatisfación Corporal para adolescentes. Revista de Saúde Pública, 43(3), 515-524. doi:10.1590/S0034-89102009000300016

Conti, M. A., Cordás, T. A., \& Latorre, M. R. D. O. (2009). A study of the validity and reliability of the Brazilian version of the Body Shape Questionnaire (BSQ) among adolescents. Revista Brasileira de Saúde Materno Infantil, 9(3), 331-338. doi:10.1590/S1519-38292009000300012

Corseuil, M. W., Pelegrini, A., Beck, C., \& Petroski, E. L. (2009). Prevalência de insatisfação com a imagem corporal e sua associação com a inadequação nutricional em adolescentes. Revista de Educação Física/UEM, 20(1), 25-31. doi:10.4025/ reveducfis.v20i1.3496

Crow, S., Eisenberg, M. E., Story, M., \& Neumark-Sztainer, D. (2008). Suicidal behavior in adolescents: Relationship to weight status, weight control behaviors, and body dissatisfaction. International Journal of Eating Disorders, 41(1), 82-87. doi:10.1002/eat.20466

Dumith, S. C., Menezes, A. M. P., Bielemann, R. M., Petresco, S., Silva, I. C. M., Linhares, R. S., Amorim, T. C., Duarte, D. V., Araújo, C. L. P., \& Santos, J. V. (2012). Insatisfação corporal em adolescentes: um estudo de base populacional. Ciência e Saúde Coletiva, 17(9), 2499-2505. doi:10.1590/S141381232012000900030

Gardner, R. M., \& Brown, D. L. (2010). Body image assessment: A review of figural drawing scales. Personality and Individual Differences, 48(2), 107-111. doi:10.1016/j. paid.2009.08.017

Gardner, R. M. (2011). Perceptual measures of body image for adolescents and adults. In T. F. Cash, \& L. Smolak (Eds.), Body image: a handbook of science, practice, and prevention (pp. 146-153). New York: The Guilford Press.

Gardner, R. M. (2012). Measurement of perceptual body image. In T. F. Cash (Ed.). Encyclopedia of body image and human appearance (pp. 526-532). Oxford: Elsevier.

Gardner, R. M., Jappe, L. M., \& Gardner, L. (2009). Development and validation of a new figural drawing scale for body-image assessment: the BIAS-BD. Journal of Clinical Psychology, 65(1), 113-122. doi:10.1002/jclp.20526

Gardner, R. M., Friedman, B. N., \& Jackson, N. A. (1998). Methodological concerns when using silhouettes to measure body image. Perceptual and Motor Skills, 86(2), 387-395. doi:10.2466/pms.1998.86.2.387
Gardner, R. M., Stark, K., Jackson, N. A., \& Friedman, B. N. (1999). Development and validation of two new scales for assessment of body-image. Perceptual and Motor Skills, 89(3), 981-993. doi:10.2466/pms.1999.89.3.981

Gray, W. N., Simon, S. L., Janicke, D. M., \& Dumont-Driscoll, M. (2011). Moderators of weight-based stigmatization among youth who are overweight and non-overweight: the role of gender, race, and body dissatisfaction. Journal of Developmental \& Behavioral Pediatrics, 32(2), 110-116. doi:10.1097/DBP.0b013e3182099754

Grogan, S. (2008). Introduction. In S. Grogan (Ed.), Body Image: understanding body dissatisfaction in men, women, and children (pp. 1-8). Londres: Routledge Publishers.

Kakeshita, I. S. (2008) Adaptação e validação de escalas de silhuetas para crianças e adultos brasileiros (Tese de doutorado), Universidade de São Paulo, Faculdade de Filosofia, Ciências e Letras de Ribeirão Preto, Brasil. Recuperado de http://www.teses.usp.br/teses/disponiveis/59/59134/tde25052008-170240/pt-br.php

Kakeshita, I. S., Silva, A. I. P., Zanatta, D. P, \& Almeida, S. S. (2009). Construção e fidedignidade teste-reteste de escalas de silhuetas brasileiras para adultos e crianças. Psicologia: Teoria e Pesquisa, 25(2), 263-270. doi:10.1590/S010237722009000200015

Kakeshita, I. S., Laus, M. F., \& Almeida, S. S. (2013). "Living well but looking good: A modern health dichotomy" A brief overview on women's body image. Motriz, 19(3), 558-564. Retrieved from http://www.periodicos.rc.biblioteca.unesp.br/ index.php/motriz/article/view/7192/pdf

Laus, M. F., Kakeshita, I. S., Braga Costa, T. M., Ferreira, M. E. C., Fortes, L. S., \& Almeida, S. S. (2013). Body image research in Brazil: recent advances in the state of knowledge and methodological issues. Aceito para publicação na Revista de Saúde Pública.

Laus, M. F, Braga Costa, T. M. B., \& Almeida, S. S. (2011). Body image dissatisfaction and its relationship with physical activity and body mass index in Brazilian adolescents. Jornal Brasileiro de Psiquiatria, 60(4), 315-320. doi:10.1590/S004720852011000400013

Menzel, J. E., Krawczyk, R., \& Thompson, J. K. (2011). Attitudinal assessment of body image for adolescents and adults. In T. F. Cash, \& L. Smolak (Eds.), Body image: a handbook of science, practice, and prevention (pp. 154-169). New York: The Guilford Press.

Ministério da Saúde. (2007). Incorporação das curvas de crescimento da Organização Mundial da Saúde de 2006 e 2007 no SISVAN. Brasília: Ministério da Saúde. Retrieved from http://189.28.128.100/nutricao/docs/geral/curvas oms_2006_2007.pdf

Pereira, E. F., Graup, S., Lopes, A. D. S., Borgatto, A. F., \& Daronco, L. S. E. (2009). Percepção da imagem corporal de crianças e adolescentes com diferentes níveis socio-econômicos na cidade de Florianópolis, Santa Catarina, Brasil. Revista Brasileira de Saúde Materno Infantil, 9(3), 253-262. doi:10.1590/S151938292009000300004

Scagliusi, F. B., Alvarenga, M., Polacow, V. O., Cordás, T. A., de Oliveira Queiroz, G. K., Coelho, D., Philippi, S. T., \& Lancha Jr., A. H. (2006). Concurrent and discriminant validity of the Stunkard's figure rating scale adapted into Portuguese. Appetite, 47(1), 77-82. doi:10.1016/j.appet.2006.02.010 
Smolak, L. (2004). Body image in children and adolescents: where do we go from here? Body Image, 1(1), 15-28. doi:10.1016/ S1740-1445(03)00008-1

Stunkard, A., Sorensen, T., \& Schlusinger, F. (1983). Use of Danish adoption register for the study of obesity and thinness. In S. Kety, L. P. Rowland, R. L. Sidman, \& S.W. Matthysse (Eds.), The genetics of neurological and psychiatric disorders (pp. 115-120). New York: Raven.

Thompson, M. A., \& Gray, J. J. (1995). Development and validation of a new body-image assessment scale. Journal of Personality Assessment, 64(2), 258-269. doi:10.1207/s15327752jpa6402_6

Thompson, J. K., \& Berg, P., van den. (2002). Measuring body image attitudes among adolescents and adults. In T. F. Cash, \& T. Pruzinsky (Eds.), Body image: a handbook of theory, research, and clinical practice (pp 142-154). New York: Guilford.

Thompson, J. K. (2004). The (mis)measurement of body image: ten strategies to improve assessment for applied and research purposes. Body Image. 1(1), 7-14. doi:10.1016/S17401445(03)00004-4

Thompson, J. K., Burke, N. L., \& Krawczyk, R. (2012). Measurement of body image in adolescence and adulthood. In T. F. Cash (Ed.), Encyclopedia of body image and human appearance (pp. 512-520). Oxford: Elsevier.
Torresani, M. E., Casós, M. E., Español, S., García, C., Salaberri, D., \& Spirito, M. F. (2009). Comparación del grado de satisfacción de la figura corporal según género en adolescentes del colegio ILSE-UBA. Diaeta, 27(128), 15-21. Retrieved from http:// www.scielo.org.ar/pdf/diaeta/v27n128/v27n128a02.pdf

Westerberg-Jacobson, J., Edlund, B., \& Ghaderi, A. (2010). A 5-year longitudinal study of the relationship between the wish to be thinner, lifestyle behaviours and disturbed eating in 9-20-year old girls. European Eating Disorders Review, 18(3), 207-219. doi:10.1002/erv.983

Xie, B., Unger, J. B., Gallaher, P., Johnson, C. A., Wu, Q., \& Chou, C. P. (2010). Overweight, body image, and depression in Asian and Hispanic adolescents. American Journal of Health Behavior, 34(4), 476. doi:10.5993/AJHB.34.4.9

Recebido em 08.12.2011

Primeira decisão editorial em 06.05.2013

Versão final em 02.07.2013

Aceito em 09.07.2013 